\title{
Development Of Human-Robot Interaction System (HRIS) Using Autonomous Programmable Humanoid Robot And Motion Sensing Input Device
}

\author{
Wan Zharfan Bin Wan Zainal Abiddin, Rozita Jailani, Abdul Rahman Omar
}

\begin{abstract}
This paper presents the development process of Human-Robot Interaction System (HRIS) based on movement imitation using autonomous programmable Humanoid Robot NAO and Microsoft Kinect Motion Sensor. This interactive platform enables autistic children and Humanoid Robot NAO to imitate each other in real time aided by Microsoft Kinect Sensor for skeletal tracking movement. Then, all the required information is uploaded to online server for therapists' evaluation. Pilot test has been performed to evaluate the performance of HRIS in imitation therapy session. The session has been tested with normal healthy children where they imitate Humanoid Robot NAO movement with reference to several different upper body posture.
\end{abstract}

Keywords-Humanoid robot, motion sensor, skeletal tracking, imitation therapy, upper body posture

\section{INTRODUCTION}

Autism is a of neurodevelopmental disorder that influences individual behavior in daily basis. Autism can be placed into three main behavior categories of social interaction, verbal and non-verbal communication impairment, and also restrictive and repetitive behavior [1]. However, it does not mean that an individual must have all of the three characteristics to be diagnosed with autism as this neurodevelopmental disorder is a collective range of disorders. Along these lines, any type of disorder which has the same qualities as the three categories can likewise be classified as autism, for example, Asperger syndrome. Statistic shows that the number of individuals with autism are increasing [2-4]. Therefore, studies are currently conducted by researchers and therapists to develop the most suitable approach to assist individuals with autism. These actions are very important to ensure the autistic person can have a normal independent life without frequent continuous supervision. Based on current research and studies, it is recommended to intervene when this type of disorder occurs from childhood period [5] as it has been proved that kid is more inclined in learning and absorbing information compared to adults [6].

This manuscript is submitted on $27^{\text {th }}$ May 2019 and accepted on $07^{\text {th }}$ January 2020. Wan Zharfan Bin Wan Zainal Abiddin and Rozita bt Jailani are with the Faculty of Electrical Engineering, Universiti Teknologi MARA, 40450 Shah Alam, Selangor (e-mail: wanzharfan92@gmail.com)

1985-5389/C 2021 The Authors. Published by UiTM Press. This is an open access article under the CC BY-NC-ND license (http://creativecommons.org/ licenses/by-nc-nd/4.0/).
Autism treatment is kept on being studied and developed from time to time. Autism is considered as a type of schizophrenia in 1976 [7]. During that period, the treatments that were being used are chelation treatment [8], electroconvulsive therapy [9], and electro-shocking [10]. Nowadays, therapy is one of the famous treatment for autistic person. Common therapy that currently being applied is the Applied Behavior Analysis (ABA) [11] where rewards are given to the autistic person when he or she displays the required behavior as a complimentary for them and for encouragement to continue doing the correct behavior.

Parallel with technology trend nowadays, implementation of current technology into autism intervention is being studied [12]. This can be explained through the nature of technology itself, as autistic children can be easily attracted to technology devices [13]. Current studies show that implementation of video interaction in social skills teaching [14] has been proposed and iPad are also being used in education of autistic child [15]. Implementation of robots in autism therapy has been recognized as utilization of robotic technologies in medical mediation is not a new thing. Research shows that anthropoid robot named Paro has been used in dementia treatment [16]. Thus, Human-Robot Interaction (HRI) that enables humanoid robot to interact with autistic children and imitation of human posture and body languages by the robot is a possible intervention for current research. Nowadays, there have been increasing interest in using humanoid robot for autism intervention [1719].

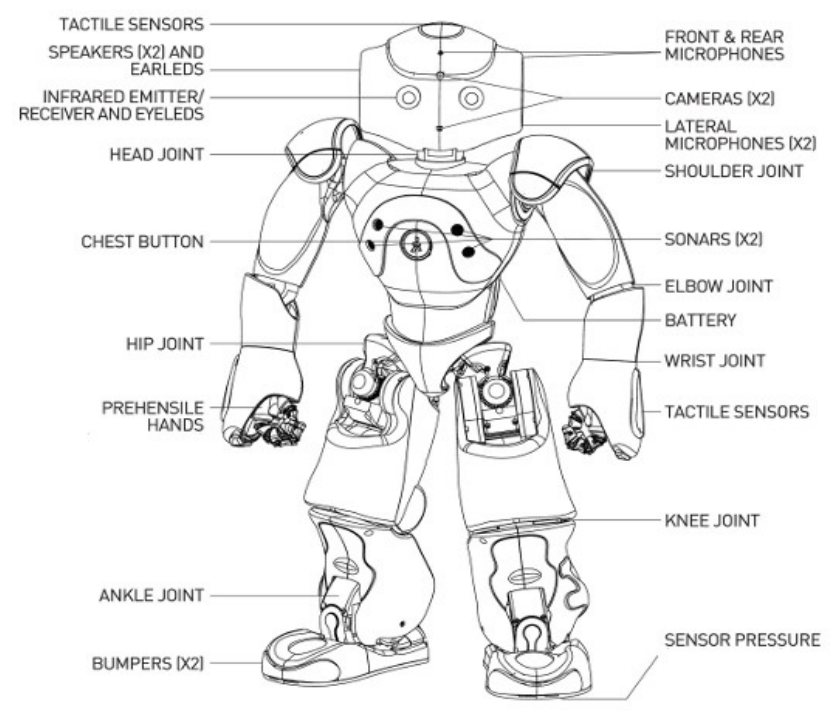

Fig. 1. Humanoid Robot NAO Specifications [20] 
Referring to Figure 1 above, humanoid robot NAO which is one of the most popular humanoid robot nowadays, made from multiple sensors in his head, hands, feet and sonars, 25 motors that controls 25 degrees of freedom, 4 directional microphones and loudspeakers, 2 cameras, 2 different connection modes of WIFI and Ethernet and operated by Naoqi OS and Choregraphe software. It is designed to be personalized in terms of capabilities, contents, personality enrichment, and new skills.

NAO has been utilized by researchers for better understanding in walking algorithm [21, 22] and intercommunication between multiple humanoid robots [23]. NAO also has been used to initiate the interactions and communications towards the autistic children [24].

Humanoid Robot NAO and Kinect Sensor have been used for iconic gestures which consist of speech and gesture integration for robotic communicator towards human [25, $26]$. Recent studies show that hand gestures improve user perceptions of robots in terms of likability, competence, and future contact intentions [27-29].

Microsoft Kinect Sensor is a tool or additional module for Microsoft Xbox which functions to track human body joints for video games. This sensor is able to track 3-D movement using its depth, RGB, and infrared sensor which detect the coordinate of 20 main body joints at $30 \mathrm{~Hz}$. It can recognize user's movement and voice commands aids by inbuilt RGB camera, infrared camera and microphone which shown in Figure 2. There are infrared projector and sensor function to capture depth images, RGB camera helps in capturing color images, multi-array microphone used to record audio as well as locating the location of sound source and direction of audio wave, and motorized tilt that tilts the sensor head up and down.

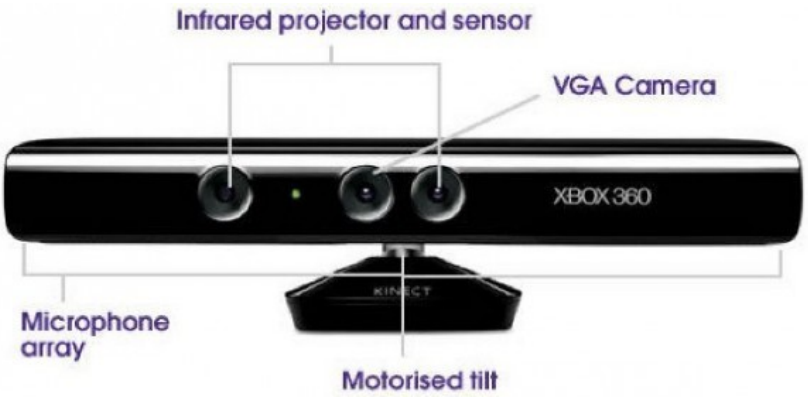

Fig. 2. Microsoft Kinect Sensor

Humanoid robot nowadays is able to represent actual person by doing human job. However, it is still hard for a humanoid robot to copy or imitate a human being since it need to see and analyze a person action in real time to imitate it. The problem can be solved by integrating 3D sensor like Microsoft Kinect Sensor which make it possible to precisely track a person behavior in real time. Recent study describes the complete forward kinematics equations and closed form inverse kinematics equations of NAO robot [30]. NAO arm has been mapped to Kinect data from actual human purposely for imitation $[31,32]$. Three different method used for NAO robot to imitate human upper body motion which are direct angle mapping method, inverse kinematics using iterative Jacobian, and inverse kinematics using fuzzy logic [33]. An interactive platform where NAO humanoid robot provides coaching towards children with autism has been developed. The framework consists of NAO humanoid robot imitates upper limb movement of autistic child aided by
Kinect sensor for skeletal tracking [34]. A motor learning and training method based on imitation and speech instruction which enables autistic children and humanoid robot NAO to imitate each other has been tested [35]. In this method, children are asked by the robot to imitate its action. Children's skeleton tracking data is captured using Kinect Sensor to evaluate the action's accuracy and consistency between human and robot. Mimicking and analyzing human motion method in real time will be developed to provoke and increase the children's imitation skills with the help of humanoid robot [36]. For the purpose of implementing and improve the learning imitation skills for autism intervention, a novel closed-loop autonomous robotic framework has been studied [37].

Therefore, in this paper, Human-Robot Interaction System (HRIS) which utilized Humanoid Robot NAO and Microsoft Kinect Sensor is proposed. Humanoid Robot NAO is able to imitate and being imitated by autistic children movement in real time aided by Microsoft Kinect Sensor for skeletal tracking movement. Then, all the required information can be uploaded to online server for therapists' evaluation.

\section{METHODOLOGY}

This project has been carried out at the Centre for Humanoid Robots \& Bio-sensing, Faculty of Mechanical Engineering, Universiti Teknologi MARA, 40450 Selangor, Malaysia. This has been tested with NAO robot V5 with Matlab software version R2015a.

\section{A. System Inplementation}

A situation is created for autistic children to communicate and interact with humanoid robot NAO assisted by Microsoft Kinect Sensor. Pilot experiment has been conducted to calculate the angle of certain body joints in real time aided by customized Matlab functions. Data is compared with ViCON motion analysis system as the point angle references [38].

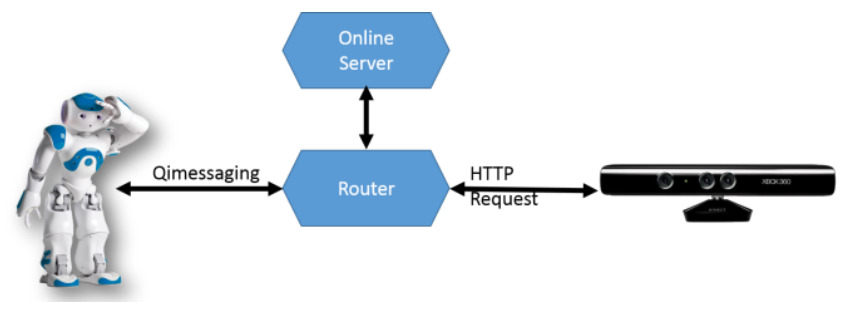

Fig. 3. Overall System Implementation

Referring to Figure 3, the system is composed of a NAO robot and Microsoft Kinect Sensor which connected wirelessly to router that is accessible through online server, with the Kinect sensor connected to a laptop computer. Within this created situation, the child can imitate robot's action and reversely the robot is able to imitate the child's action aided by Kinect sensor in real time. All information is then stored in server database for patient monitoring progress and evaluation.

Figure 4 shows the conceptual framework of this project development. Program starts and a graphical user interface (GUI) appears. Then, a connection is established to Humanoid NAO Robot through HTTP request. If connection is successful, NAO module selection interface appears indicating that the robot is ready to be operated. User needs 
to choose a module for the operational task. Then, NAO will perform the selected scenario assisted by Microsoft Kinect Sensor for movement calculation. After that, when the operational module has ended, a GUI based evaluation form needed to be filled by parent or user to evaluate the therapy session that has been done. Evaluation form is based on a recent study that has been conducted [39]. Then, the evaluation data are uploaded to online server database along with MATLAB data for therapists' evaluation and monitoring purpose.

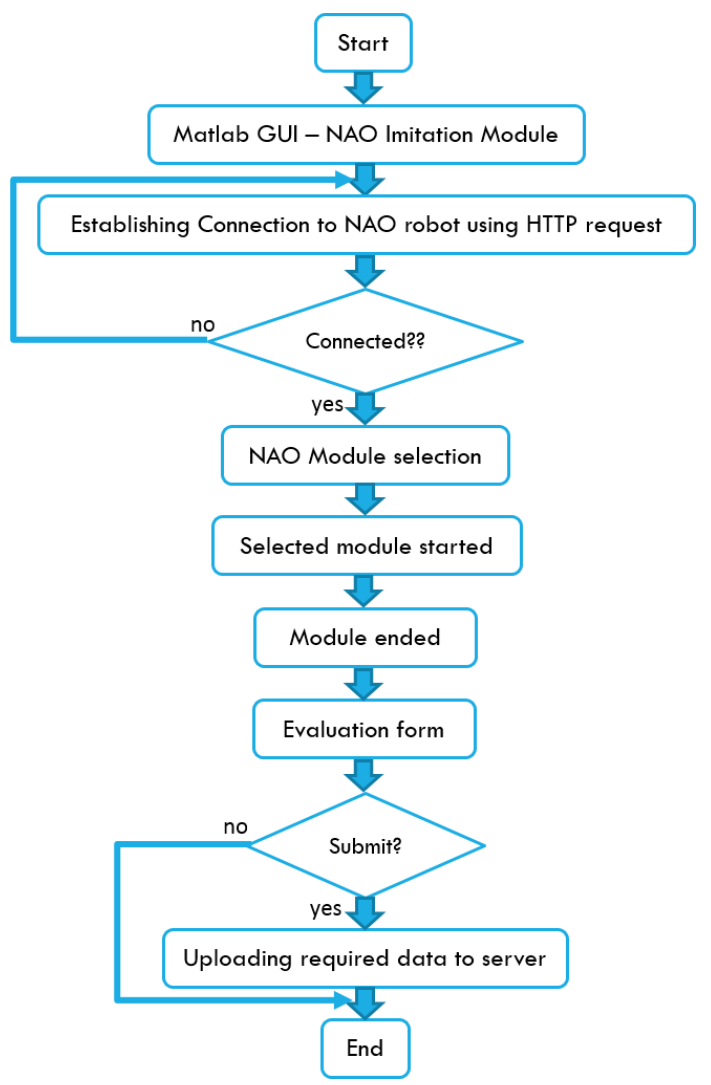

Fig. 4. Conceptual Framework

\section{B. Interfacing NAO with Local Server}

Humanoid robot NAO is connected to a router and accessible through online server. The server stores all the JavaScript files which are required to control the behavior of NAO robot using QiMessaging method. QiMessaging is another NAO architecture, permitting the use of NAOqi APIs with a simpler and easier code structure [40]. NAOqi is the name of the primary software which runs on the robot and controls it. In this project, Qimessaging enables JavaSript bindings to use Qimessaging modules in a web program which permits the HTML5 web application for NAO robot [41].

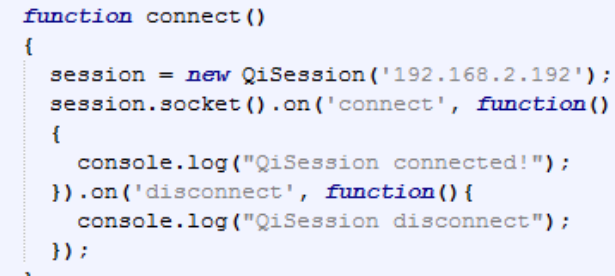

Fig. 5. JavaScript Bindings
Figure 5 shows some scripting for interfacing Humanoid robot NAO with web application. The script hosted on server requires the inclusion of Qimessaging which is facilitated on the robot. The bindings provide only one class of QiSession which interfaces with robot NAO and acquires proxies to services. The connection requires the hostname or IP address of the robot.

\section{Human's Motion Sensing and Recognition}

Microsoft Kinect Sensor is used to record skeletal tracking data of autistic children and it is attached on tripod stand as shown in Figure 6. The device is positioned in front of the child for skeletal tracking while humanoid robot NAO is standing near the tripod stand. The device was connected to a USB port of laptop running Windows 764 bit. Data from Kinect Sensor was extracted to MATLAB software using Image Acquisition Toolbox for Kinect Sensor and Kinect for Windows SDK.

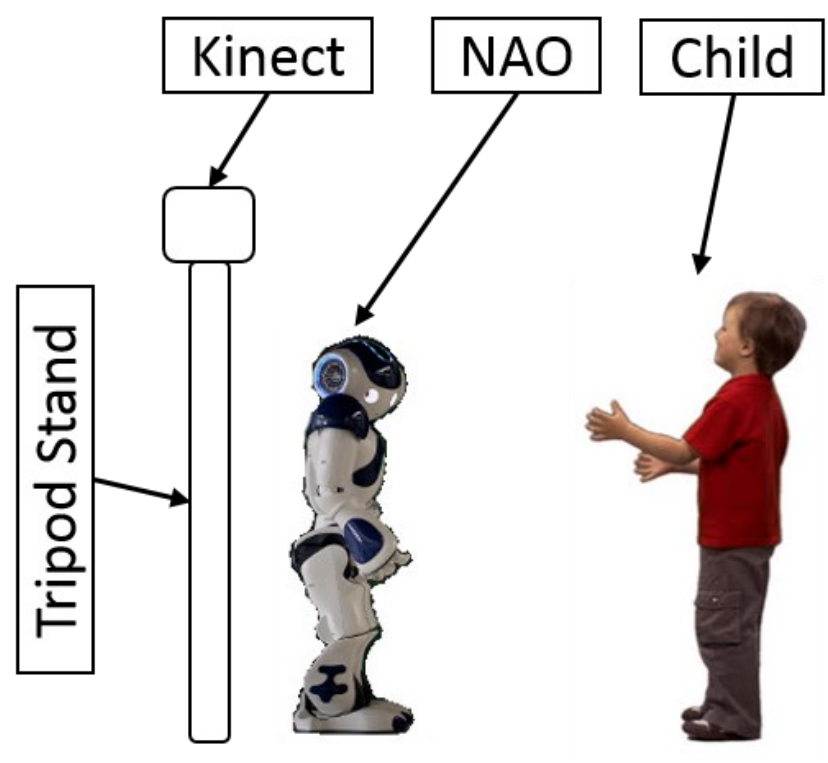

Fig. 6. Experimental Setup

MATLAB code was customized and programmed to recognize skeletal movement of 20 main body joints coordinates as shown in Figure 7. In this experiment, only five body joints are tracked and recorded with Kinect Sensor. Five body joints are right elbow, right shoulder, spine, left shoulder, and left elbow. These coordinate of body joints are compulsory to measure the required angle used in this experiment. 


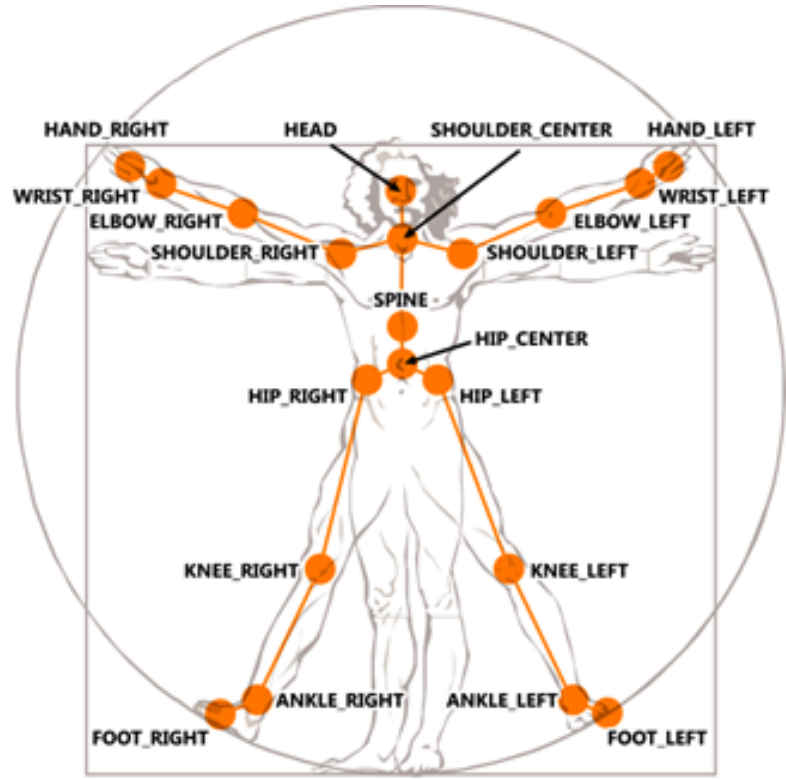

Fig. 7. 20 main body joints [42]

A customized function [38] was added to calculate the angle of required joints for NAO Imitation Module. The calculations are based on the pixel coordinates of the body joints as shown in Figure 8.

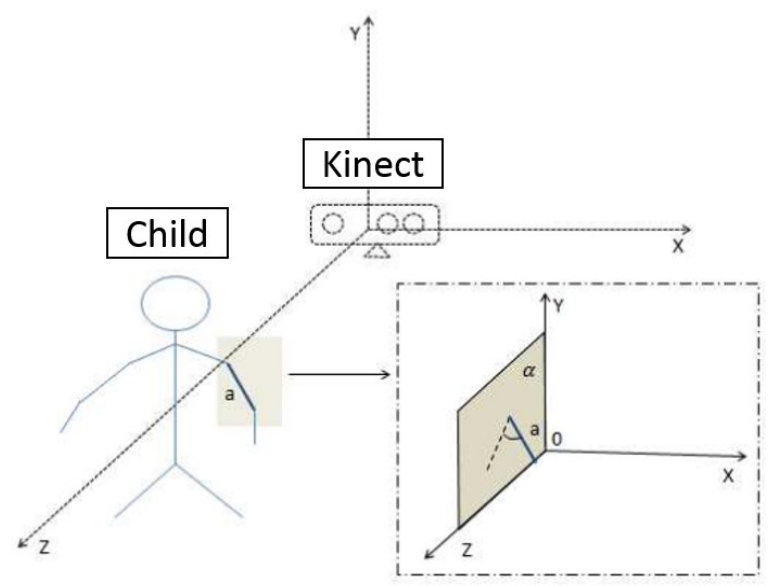

Fig. 8. Pixel coordinates of body joints

Each coordinate of body joints is in a three dimensional coordinate which the origin is the Kinect Sensor. Y-axis indicates the upright and downright position of the coordinates, $\mathrm{X}$-axis refers to the left and right position of the body joints, while $\mathrm{Z}$-axis shows the joints position straight from the Kinect Sensor. X-coordinates and $\mathrm{Y}$-coordinates are used in measuring the required body joint angles while $\mathrm{Z}$ coordinates are used to identify the distance of child from the Kinect Sensor. The distance measurement is important to make sure the patient is in the right position in front of Kinect Sensor. Angle measurement only will be triggered when the patient is in the required range of distance. The calculated angles are then used to compare the similarity of motion between the child and NAO robot.

\section{Behavior of NAO robot}

NAO robot behavior is controlled through local server assisted by Kinect Sensor. A series of motions and postures are chosen as NAO Imitation Module. The module is developed based on these 2 criteria: -
1. The motions are simple enough for autistic children to imitate

2. The motions can be accurately captured by Kinect sensor

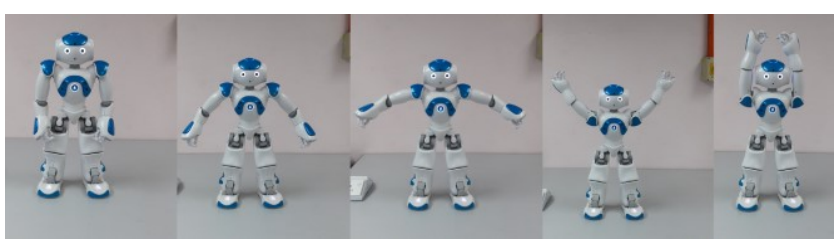

Fig. 9. Humanoid Robot NAO posture

Based on these criteria, NAO robot is programmed to make these poses as illustrated in Figure 9, then the corresponding actions are imitated by autistic children as the skeletal data is captured by Kinect sensor to match those posture. The first picture indicates 'Standing Pose', second picture shows 'Fathoms Hands Down Pose', third picture illustrates 'Fathoms Hands Pose', fourth picture shows 'Fathoms Hands Up Pose' and the last picture shows 'Hands Up Pose'.

\section{E. NAO Imitation Module}

Figure 10 shows the process and steps for a child to imitate NAO robot. First, NAO will make a pose or posture and instruct the child to imitate the movement. After that Kinect sensor will acquire the required child's angle data for a short time period. If the angle data is in the range of requirement which indicating the child success in imitating the pose, a praise reward is given such for the successful imitation. Then, NAO returns to standing position.

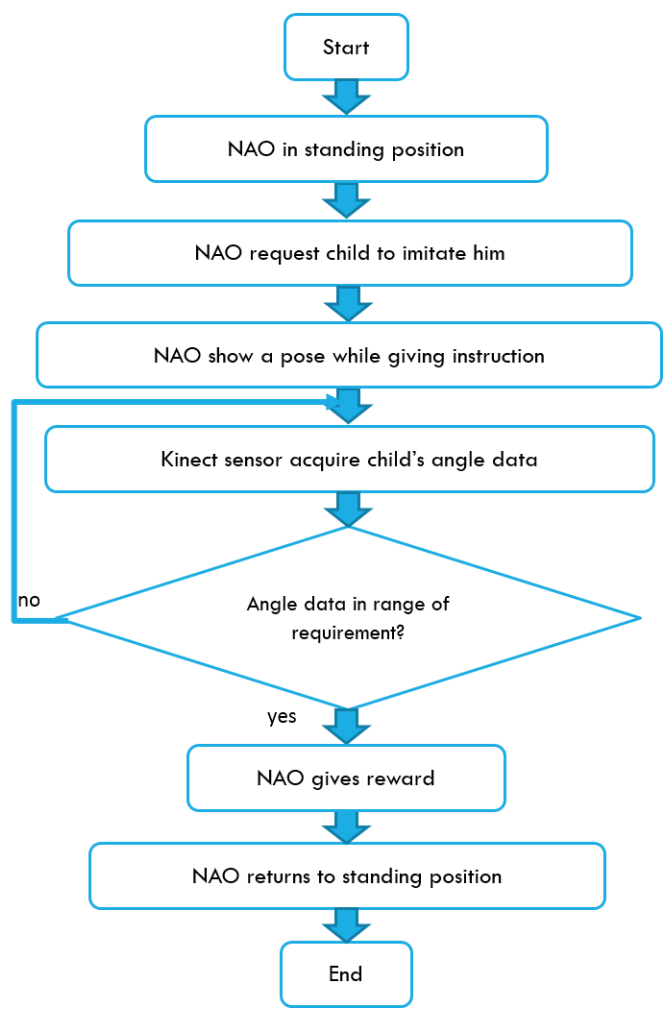

Fig. 10. Child Imitates Robot Flowchart 


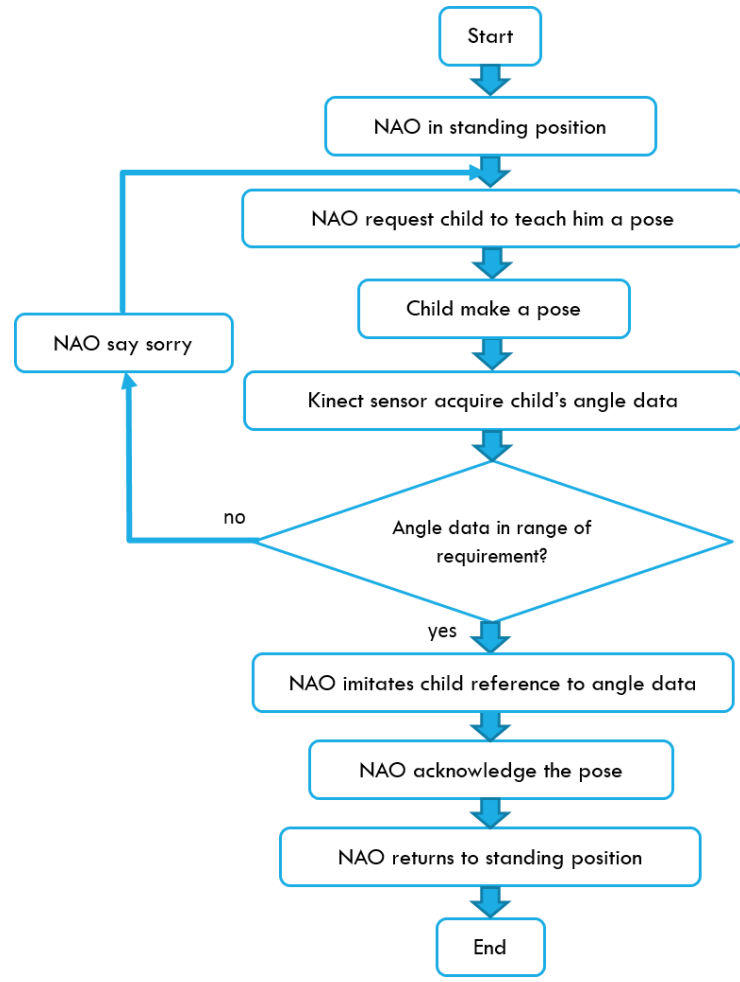

Fig. 11. Robot Imitates Child Flowchart

Figure 11 shows the process and steps for NAO to imitates child's simple action. First, NAO in standing position. Then, the robot requests the child to do a pose. Then, the child will do a simple pose in 5 seconds period. After that, Kinect Sensor capture the required angle's data. If the data is in the range of requirement which indicating possible action for NAO to imitate, the robot will make that pose with reference to the captured angle and says "I completed the task" and return to standing position. If the motion is impossible for the robot to imitate, NAO will stand still and say "I am sorry, I can't do that pose" and request the child to do another pose.

\section{RESULTS AND DISCUSSION}

This project paper focused on the development of Human-Robot Interaction System (HRIS) using Humanoid Robot NAO to imitate or being imitate by autistic children with the help of Microsoft Kinect Sensor as children's motion sensing and recognition. This paper differs is a continuation from previous work [43]. Previous work focused on development of human-robot imitation program. Current work focus on development of child imitates robot and robot imitates child module. Results contribute to system testing on subjects for child imitates robot module with all the required information is uploaded to online database for therapists' evaluation as shown in Table I below.

TABLE I

Child Imitates Robot Module Result In OnLINE Database

\begin{tabular}{|c|c|c|c|c|c|c|c|}
\hline ID & Timestamp & P1 & P2 & P3 & P4 & P5 & Score \\
\hline $\mathbf{1}$ & $\begin{array}{c}2018-05-23 \\
10: 56: 18\end{array}$ & 3 & 3 & 3 & 3 & 3 & 15 \\
\hline $\mathbf{2}$ & $\begin{array}{c}2018-05-23 \\
11: 10: 47\end{array}$ & 3 & 2 & 2 & 3 & 2 & 12 \\
\hline $\mathbf{3}$ & $\begin{array}{c}2018-05-23 \\
11: 28: 23\end{array}$ & 3 & 1 & 2 & 1 & 2 & 9 \\
\hline $\mathbf{4}$ & $2018-05-23$ & 3 & 3 & 1 & 3 & 2 & 12 \\
\hline
\end{tabular}

\begin{tabular}{|l|l|l|l|l|l|l|l|}
\hline & $11: 56: 18$ & & & & & & \\
\hline
\end{tabular}

Table I shows the child imitates robot module results stored in online database for therapists' evaluation. The column "ID" is for the unique id of the patient, "TimeStamp" is for the time and date of the data being uploaded, "P1" is for the number of trials for "Standing Pose", "P2" is for the "Fathoms Hands Down Pose", "P3" is for the "Fathoms Hands Pose", "P4" is for the "Fathoms Hands Up Pose", and lastly "P5" column is for "Hands Up Pose". The marks given are based on the number of trial they achieve for the correct pose as demonstrated by humanoid robot NAO. If the patient fail to imitate the pose within three trial, result ' 0 ' will be given. If the patient succeeded in the third trial, ' 1 ' will be given for the result, "2" will be given if the imitation is correct on the second trial and lastly, " 3 " will be given if the imitation is correct on the first trial. Duration of each trial is set to five seconds after each pose instruction was given.

TABLE II

RIGHT SHOULDER JOINT ANGLES

\begin{tabular}{|c|c|c|c|c|c|}
\hline \multirow{2}{*}{ Subject } & \multicolumn{5}{|c|}{ Right Shoulder Joint Angles } \\
\cline { 2 - 6 } & Pose 1 & Pose 2 & Pose 3 & Pose 4 & Pose 5 \\
\hline Subject 1 & 53 & 74 & 100 & 182 & 231 \\
\hline Subject 2 & 50 & 70 & 102 & 186 & 219 \\
\hline Subject 3 & 48 & 68 & 99 & 190 & 220 \\
\hline Subject 4 & 50 & 69 & 94 & 192 & 225 \\
\hline Subject 5 & 52 & 70 & 95 & 186 & 222 \\
\hline
\end{tabular}

TABLE III

LEFT SHOULDER JOINT ANGLES

\begin{tabular}{|c|c|c|c|c|c|}
\hline \multirow{2}{*}{ Subject } & \multicolumn{5}{|c|}{ Left Shoulder Joint Angles } \\
\cline { 2 - 6 } & Pose 1 & Pose 2 & Pose 3 & Pose 4 & Pose 5 \\
\hline Subject 1 & 52 & 70 & 100 & 190 & 220 \\
\hline Subject 2 & 51 & 68 & 98 & 186 & 219 \\
\hline Subject 3 & 50 & 68 & 97 & 184 & 230 \\
\hline Subject 4 & 49 & 72 & 103 & 187 & 222 \\
\hline Subject 5 & 51 & 73 & 94 & 190 & 218 \\
\hline
\end{tabular}

An experiment is conducted to test Child Imitates Robot Module. With reference to previous experiment [38], five healthy children volunteered for this project. They were asked to imitate NAO robot's action for five different poses. Right shoulder and left shoulder joint angles for each of their poses were calculated and recorded as shown in Table II and Table III. 'Pose 1' indicates 'Standing Pose', 'Pose 2' shows 'Fathoms Hands Down Pose', 'Pose 3' illustrates 'Fathoms Hands Pose', 'Pose 4' shows 'Fathoms Hands Up Pose' and the last 'Pose 5' shows 'Hands Up Pose'. For right shoulder joint angles, joint angle for Pose 1 range from 48 to 53, Pose 2 range from 68 to 74, Pose 3 range from 94 to 102, Pose 4 range from 182 to 192 , while Pose 5 range from 219 to 231 . For left shoulder joint angles, joint angle for Pose 1 range from 49 to 52, Pose 2 range from 68 to 73, Pose 3 range from 94 to 103 , Pose 4 range from 184 to 190 , while Pose 5 range from 218 to 230 . 


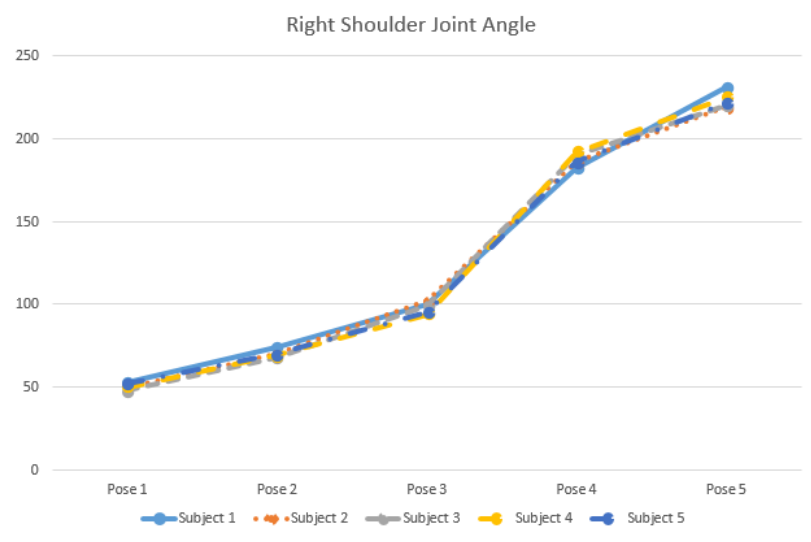

Fig. 13. Graph of right shoulder angle among tested subject for each different pose

Left Shoulder Joint Angle

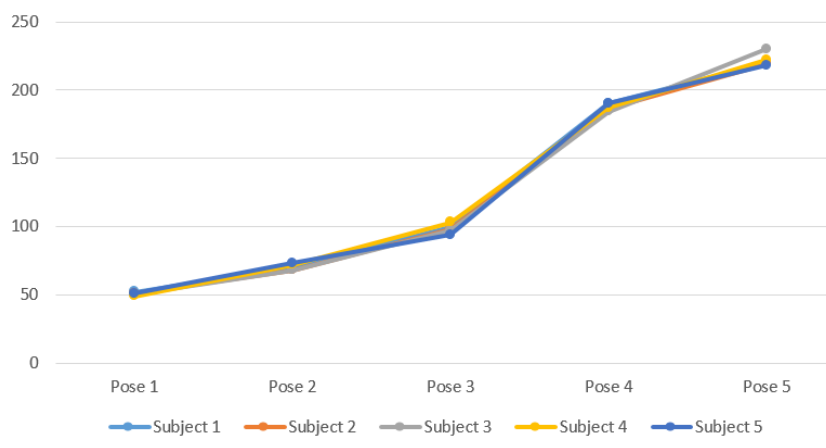

Fig. 14. Graph of left shoulder angle among tested subject for each different pose

Referring to the graph pattern in Figure 13 and Figure 14, angle distribution of each pose is concentrated for each subject. It can be concluded that HRIS can track joint coordinates consistently for human movement tracking which in this experiment, five body joints coordinates are used for right and left shoulder angle measurement.

Based on angle result for each pose for five different subjects in Table II, Table III, Figure 13 and Figure 14, a range sets of angles are determined to identify and validate each pose for results shown in Table I. Range sets of angles for left and right shoulders for each pose is presented in Table IV. Range sets of joint angles for Pose 1 range from 48 to 53, Pose 2 range from 68 to 74, Pose 3 range from 94 to 103, Pose 4 range from 182 to 192, while Pose 5 range from 219 to 231 .

TABLE IV

RANGE SETS OF ANGLES FOR LEFT AND RIGHT SHOULDERS

\begin{tabular}{|c|c|}
\hline Subject & $\begin{array}{c}\text { Range Sets of Angles for } \\
\text { Left and Right Shoulders }\end{array}$ \\
\hline Pose 1 & $48-53$ \\
\hline Pose 2 & $68-74$ \\
\hline Pose 3 & $94-103$ \\
\hline Pose 4 & $182-192$ \\
\hline Pose 5 & $219-231$ \\
\hline
\end{tabular}

Test on normal subject proved that HRIS can be used for imitation session. However, there were few limitations of this study which need to be imposed. The test subjects used in this research were healthy children. It is not confirmed whether the angle readings or the graph pattern will be the same if it is tested on the autistic children. It remains unclear whether HRIS is good enough to measure the difference in shoulder joint angle of autistic children. Results will be depending on how well the children can perform the required task during therapy session. Therefore, further studies must be taken to consideration to study the relative comparison between healthy and autistic children using HRIS to differentiate the performance of HRIS for autism therapy session.

Based on studies, there are a few indicators which significantly affect the performance of HRIS. Accuracy of angle reading measurement is different with reference to the movement speed. Previous studies indicate that Microsoft Kinect Sensor is able to track human motion with small error in coordinate measurement depending on movement speed, which is related to the speed of captured frames.

\section{CONCLUSION}

Previous studies highlighted that imitation aided by humanoid robot can improve the social skills of autistic children. An interactive autism therapy session using Humanoid Robot NAO and Microsoft Kinect Sensor based on imitation movement is developed to improve the social skills of autistic children. It has been tested with 5 normal children and will be further studied and tested on children with autism. Results indicate that HRIS successful in conducting imitation session. Results gathered from the test will be investigated and analyzed to examine the performance and effectiveness of HRIS as autism therapy session.

\section{ACKNOWLEDGMENT}

An acknowledgement is accorded to the Ministry of Education, Malaysia for the funds received through BESTARI Grant Scheme, Project file: 600-IRMI/MyRA 5/3/BESTARI (020/2017) and to the Centre for Humanoid Robots \& Bio-sensing, Faculty of Mechanical Engineering, Universiti Teknologi MARA (UiTM) Shah Alam, 40450 Selangor, Malaysia.

\section{REFERENCES}

[1] J. A. Worley and J. L. Matson, "Comparing symptoms of autism spectrum disorders using the current DSM-IV-TR diagnostic criteria and the proposed DSM-V diagnostic criteria," Research in Autism Spectrum Disorders, vol. 6, pp. 965-970, 2012.

[2] L. Waterhouse, "Autism overflows: increasing prevalence and proliferating theories," Neuropsychology Review, vol. 18, pp. 273286, 2008.

[3] J. L. Matson and A. M. Kozlowski, "The increasing prevalence of autism spectrum disorders," Research in Autism Spectrum Disorders, vol. 5, pp. 418-425, 2011

[4] Baio, J., Wiggins, L., Christensen, D., Maenner, M., Daniels, J., Warren, Z., Kurzius-Spencer, M., Zahorodny, W., Robinson, C., Rosenberg, White, T., Durkin, M., Imm, P., Nikolaou, L., YearginAllsopp, M., Lee, L., Harrington, R., Lopez, M., Fitzgerald, R., Hewitt, A., Pettygrove, S., Constantino, J., Vehorn, A., Shenouda, J., Hall-Lande, J., Van, K., Naarden, Braun and Dowling, N. (2018). Prevalence of Autism Spectrum Disorder Among Children Aged 8 Years - Autism and Developmental Disabilities Monitoring Network, 11 Sites, United States, 2014. MMWR. Surveillance Summaries, 67(6), pp.1-23.

[5] M. Rivard, A. Terroux, and C. Mercier, "Effectiveness of early behavioral intervention in public and mainstream settings: The case of 
preschool-age children with autism spectrum disorders," Research in Autism Spectrum Disorders, vol. 8, pp. 1031-1043, 2014.

[6] M. V. Johnston, "Plasticity in the developing brain: implications for rehabilitation," Developmental disabilities research reviews, vol. 15, pp. 94-101, 2009.

[7] L. Wing, "The History of Ideas on Autism Legends, Myths and Reality," Autism, vol. 1, pp. 13-23, 1997.

[8] T. N. Davis, M. O'Reilly, S. Kang, R. Lang, M. Rispoli, J. Sigafoos, et al., "Chelation treatment for autism spectrum disorders: A systematic review," Research in Autism Spectrum Disorders, vol. 7, pp. 49-55, 2013.

[9] L. E. Wachtel, R. Jaffe, and C. H. Kellner, "Electroconvulsive therapy for psychotropic-refractory bipolar affective disorder and severe selfinjury and aggression in an 11-year-old autistic boy," European child \& adolescent psychiatry, vol. 20, pp. 147-152, 2011.

[10] D. C. Lerman, L. R. Addison, and T. Kodak, "A PRELIMINARY ANALYSIS OF SELF - CONTROL WITH AVERSIVE EVENTS: THE EFFECTS OF TASK MAGNITUDE AND DELAY ON THE CHOICES OF CHILDREN WITH AUTISM," Journal of applied behavior analysis, vol. 39, pp. 227-232, 2006.

[11] J. L. Matson, N. C. Turygin, J. Beighley, R. Rieske, K. Tureck, and M. L. Matson, "Applied behavior analysis in Autism Spectrum Disorders: Recent developments, strengths, and pitfalls," Research in Autism Spectrum Disorders, vol. 6, pp. 144-150, 2012.

[12] S. Bölte, O. Golan, M. S. Goodwin, and L. Zwaigenbaum, "What can innovative technologies do for autism spectrum disorders?" 2010.

[13] T. R. Goldsmith and L. A. LeBlanc, "Use of technology in interventions for children with autism," Journal of Early and Intensive Behavior Intervention, vol. 1, p. 166, 2004.

[14] M. Sherer, K. L. Pierce, S. Paredes, K. L. Kisacky, B. Ingersoll, and L. Schreibman, "Enhancing Conversation Skills in Children with Autism Via Video Technology Which Is Better,"Self" or "Other" as a Model?," Behavior modification, vol. 25, pp. 140-158, 2001.

[15] C. Doenyas, E. Şimdi, E. Ç. Özcan, Z. Çataltepe, and B. Birkan, "Autism and tablet computers in Turkey: Teaching picture sequencing skills via a web-based iPad application," International Journal of Child-Computer Interaction, vol. 2, pp. 60-71, 2014.

[16] W.-L. Chang, S. Šabanovic, and L. Huber, "Use of seal-like robot PARO in sensory group therapy for older adults with dementia," in Proceedings of the 8th ACM/IEEE international conference on Human-robot interaction, 2013, pp. 101-102.

[17] B. Robins, P. Dickerson, P. Stribling, and K. Dautenhahn, "Robotmediated joint attention in children with autism: A case study in robot-human interaction," Interaction studies, vol. 5, pp. 161-198, 2004.

[18] K. Dautenhahn and I. Werry, "Towards interactive robots in autism therapy: Background, motivation and challenges," Pragmatics \& Cognition, vol. 12, pp. 1-35, 2004.

[19] J.-J. Cabibihan, H. Javed, M. Ang Jr, and S. M. Aljunied, "Why robots? A survey on the roles and benefits of social robots in the therapy of children with autism," International journal of social robotics, vol. 5, pp. 593-618, 2013.

[20] A. Robotics. (2016-03-15). Find out more about NAO. Available: https://www.aldebaran.com/en/cool-robots/nao/find-out-more-aboutnao

[21] A. D. Ames, E. A. Cousineau, and M. J. Powell, "Dynamically stable bipedal robotic walking with NAO via human-inspired hybrid zero dynamics," in Proceedings of the 15th ACM international conference on Hybrid Systems: Computation and Control, 2012, pp. 135-144.

[22] J. J. Alcaraz-Jiménez, D. Herrero-Pérez, and H. Martínez-Barberá, "Robust feedback control of ZMP-based gait for the humanoid robot Nao," The International Journal of Robotics Research, vol. 32, pp. 1074-1088, 2013.

[23] T. Liu and M. Q.-H. Meng, "Study on cooperation between humanoid robot Nao and Barrett WAM," in Robotics and Biomimetics (ROBIO), 2012 IEEE International Conference on, 2012, pp. 980983.

[24] S. Shamsuddin, H. Yussof, L. I. Ismail, S. Mohamed, F. A. Hanapiah, and N. I. Zahari, "Initial response in HRI-a case study on evaluation of child with autism spectrum disorders interacting with a humanoid robot Nao," Procedia Engineering, vol. 41, pp. 1448-1455, 2012.
[25] P. Bremner and U. Leonards, "Efficiency of speech and iconic gesture integration for robotic and human communicators-a direct comparison," in 2015 IEEE International Conference on Robotics and Automation (ICRA), 2015, pp. 1999-2006.

[26] G. Canal, C. Angulo, and S. Escalera, "Gesture based human multirobot interaction," in 2015 International Joint Conference on Neural Networks (IJCNN), 2015, pp. 1-8.

[27] J. Han, N. Campbell, K. Jokinen, and G. Wilcock, "Investigating the use of non-verbal cues in human-robot interaction with a Nao robot," in Cognitive Infocommunications (CogInfoCom), 2012 IEEE 3rd International Conference on, 2012, pp. 679-683.

[28] A. Aly and A. Tapus, "A model for synthesizing a combined verbal and nonverbal behavior based on personality traits in human-robot interaction," in Proceedings of the 8th ACM/IEEE international conference on Human-robot interaction, 2013, pp. 325-332.

[29] M. Salem, F. Eyssel, K. Rohlfing, S. Kopp, and F. Joublin, "To err is human (-like): Effects of robot gesture on perceived anthropomorphism and likability," International Journal of Social Robotics, vol. 5, pp. 313-323, 2013.

[30] N. Kofinas, E. Orfanoudakis, and M. G. Lagoudakis, "Complete analytical inverse kinematics for NAO," in Autonomous Robot Systems (Robotica), 2013 13th International Conference on, 2013, pp. $1-6$.

[31] A. R. Ibrahim and W. Adiprawita, "Analytical upper body human motion transfer to naohumanoid robot," International Journal on Electrical Engineering and Informatics, vol. 4, p. 563, 2012.

[32] K.-M. Koo, Y. J. Kim, and Y.-H. Seo, "Motion capture based dual arm control of a humanoid robot using kinect," in Information Technology Convergence, ed: Springer, 2013, pp. 913-921.

[33] S. Mukherjee, D. Paramkusam, and S. K. Dwivedy, "Inverse kinematics of a NAO humanoid robot using kinect to track and imitate human motion," in Robotics, Automation, Control and Embedded Systems (RACE), 2015 International Conference on, 2015, pp. 1-7.

[34] X. Liu, C. Liu, X. Zhou, X. Zhou, and A. Jiang, "Movement imitation underlying coaching platform for children with ASD," in Consumer Electronics-Taiwan (ICCE-TW), 2015 IEEE International Conference on, 2015, pp. 57-58

[35] X. Liu, X. Zhou, C. Liu, J. Wang, X. Zhou, N. Xu, et al., "An interactive training system of motor learning by imitation and speech instructions for children with autism," in Human System Interactions (HSI), 2016 9th International Conference on, 2016, pp. 56-61.

[36] J. Greczek, E. Kaszubski, A. Atrash, and M. Matarić, "Graded cueing feedback in robot-mediated imitation practice for children with autism spectrum disorders," in The 23rd IEEE International Symposium on Robot and Human Interactive Communication, 2014, pp. 561-566.

[37] Z. Zheng, S. Das, E. M. Young, A. Swanson, Z. Warren, and N. Sarkar, "Autonomous robot-mediated imitation learning for children with autism," in 2014 IEEE International Conference on Robotics and Automation (ICRA), 2014, pp. 2707-2712.

[38] W. Z. W. Z. Abiddin, R. Jailani, A. R. Omar, and I. M. Yassin, "Development of MATLAB Kinect Skeletal Tracking System (MKSTS) for gait analysis," in 2016 IEEE Symposium on Computer Applications \& Industrial Electronics (ISCAIE), 2016, pp. 216-220.

[39] S. Shamsuddin, H. Yussof, S. Mohamed, F. A. Hanapiah, and H. A. Ainudin, "Telerehabilitation Service with a Robot for Autism Intervention," Procedia Computer Science, vol. 76, pp. 349-354, 2015.

[40] Aldebaran. (2016-09-19). Experimental: qimessaging API. Available: http://doc.aldebaran.com/2-1/dev/python/qimessaging.html

[41] Aldebaran. (2016-03-15). QiMessaging JavaScript 1.0. Available: http://doc.aldebaran.com/2-4/dev/js/index-1.0.html\#js-1-0

[42] Microsoft. (2016, 2016-03-15). Tracking Users with Kinect Skeletal Tracking. Available: https://msdn.microsoft.com/enus/library/jj131025.aspx

[43] Abiddin, W. Z. B. W. Z., Jailani, R., \& Omar, A. R. (2018, September). Development of Robot-Human Imitation Program for Telerehabilitation System. In 2018 11th International Conference on Developments in eSystems Engineering (DeSE) (pp. 198-201). IEEE. 